\title{
A Showcase of Analytical Techniques: Native V in Hibonite
}

\author{
Sarah E.M. Gain ${ }^{1,2 *}$, William L. Griffin ${ }^{2}$, Martin Saunders ${ }^{1}$, Jeremy Shaw ${ }^{1}$ and Vered Toledo ${ }^{3}$ \\ ${ }^{1}$ Centre for Microscopy, Characterisation and Analysis, The University of Western Australia, \\ WA 6009, Australia. \\ ${ }^{2}$ ARC Centre of Excellence for Core to Crust Fluid Systems (CCFS) and Centre for \\ Geochemical Evolution and Metallogeny of Continents (GEMOC), Earth and Planetary \\ Sciences, Macquarie University, NSW 2109, Australia. \\ ${ }^{3}$ Shefa Yamim (A.T.M.) Ltd., Netanya 4210602, Israel. \\ * Corresponding author: sarah.gain@uwa.edu.au
}

Hibonite $\left(\mathrm{CaAl}_{12} \mathrm{O}_{19}\right)$ is one of the earliest phases to condense from the solar nebula. It can also be found in high-temperature calc-silicate metamorphic rocks, but this is the first report of its crystallization from an igneous melt. We have found it intergrown with corundum, glass, grossite $\left(\mathrm{CaAl}_{4} \mathrm{O}_{7}\right)$, vanadium-bearing spinel [1] and native vanadium in ejecta from Cretaceous volcanoes on Mt Carmel, Israel [2,3]. The presence of $\mathrm{V}^{0}$ implies very low oxygen fugacity, similar to nebular conditions.

To understand the genesis of this unusual material, a wide range of techniques were used to study both the major minerals and their inclusions. These techniques include stereo microscopy for general characterization (Fig. 1); backscattered electron (BSE) imaging on a scanning electron microscope (SEM); energy dispersive X-ray spectroscopy (SEM-EDS) to determine mineral compositions; electron microprobe (EMP) to determine mineral chemistry; focused ion beam (FIB) for sample preparation; transmission electron microscopy (TEM) to observe submicronscale structural features and crystallography; electron energy loss spectroscopy (EELS) to determine element valences; and Micro-Computed Tomography (3D- $\mu \mathrm{CT})$ to image vanadium structures in 3D (Fig. 2).

The images reveal that the vanadium occurs both as rounded to drop-shaped inclusions, and as dendritic arrays of blobs and fibres that appear to have nucleated first on crystal faces, then continued to grow as the crystal face moved out into a surrounding melt/fluid. Pauses in crystallization appear as planes cutting off the dendrites, and new nucleation points appear on these planes, growing further with the next pulse of crystallisation. These images reveal much about the growth environment, and the detailed mechanisms and implications of dendritic crystallisation. The extremely low oxygen fugacity implies the presence of hydrogen-rich fluids, which may occupy the void spaces visible in the $3 \mathrm{D}-\mu \mathrm{CT}$ images. This suggestion is supported by the discovery of vanadium dihydride $\left(\mathrm{VH}_{2}\right)$ among the vanadium balls [4].

\section{References}

[1] F. Camara et al., Minerals 9 (2019) online.

[2] W.L. Griffin et al., Mineralogy and Petrology 112 (2018), p. 101-114.

[3] W.L. Griffin et al., American Mineralogist 104 (2019), p. 207-219.

[4] L. Bindi et al., American Mineralogist (in press, 4/2019) 


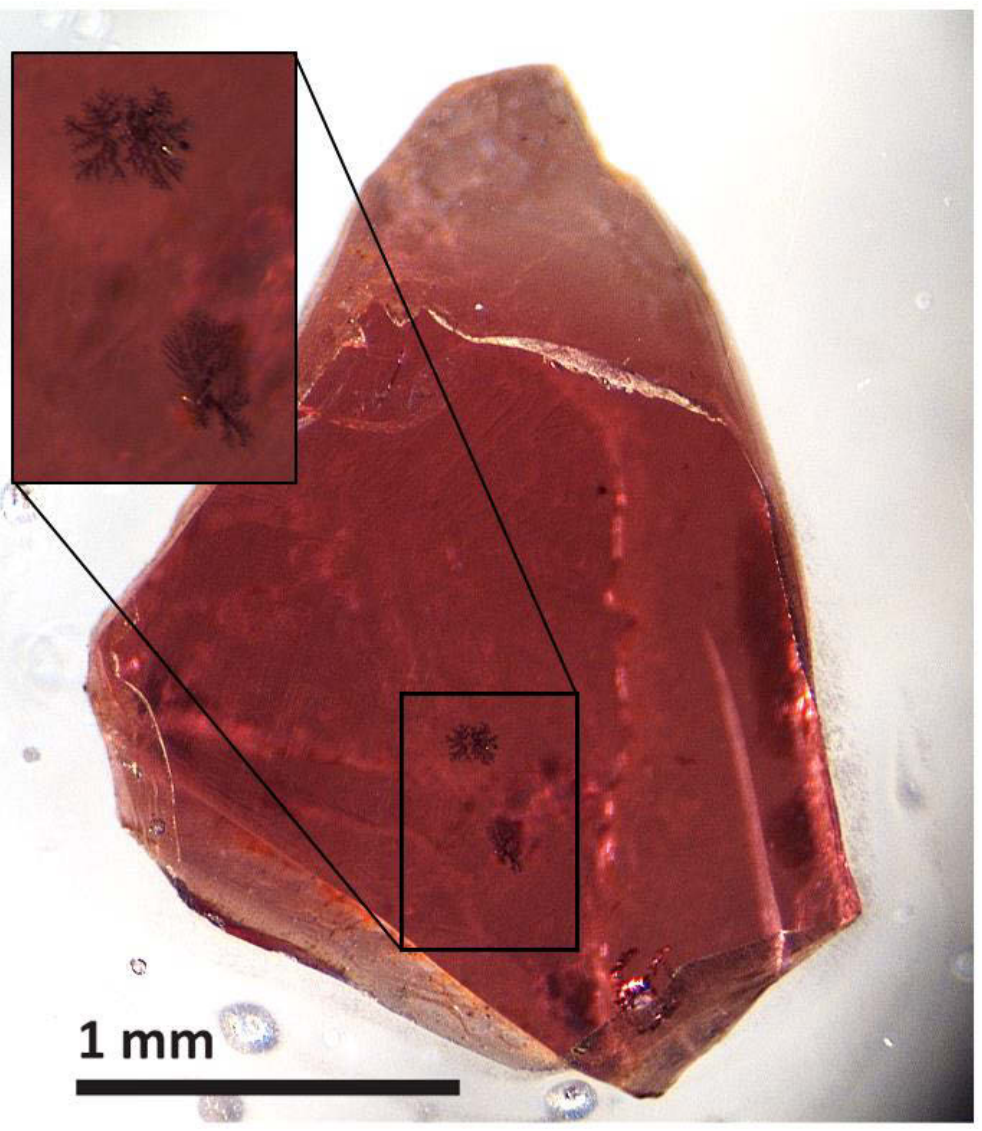

Figure 1. Stereo microscope image of hibonite with $\mathrm{V}_{0}$ inclusions (dark aggregates, see inset).
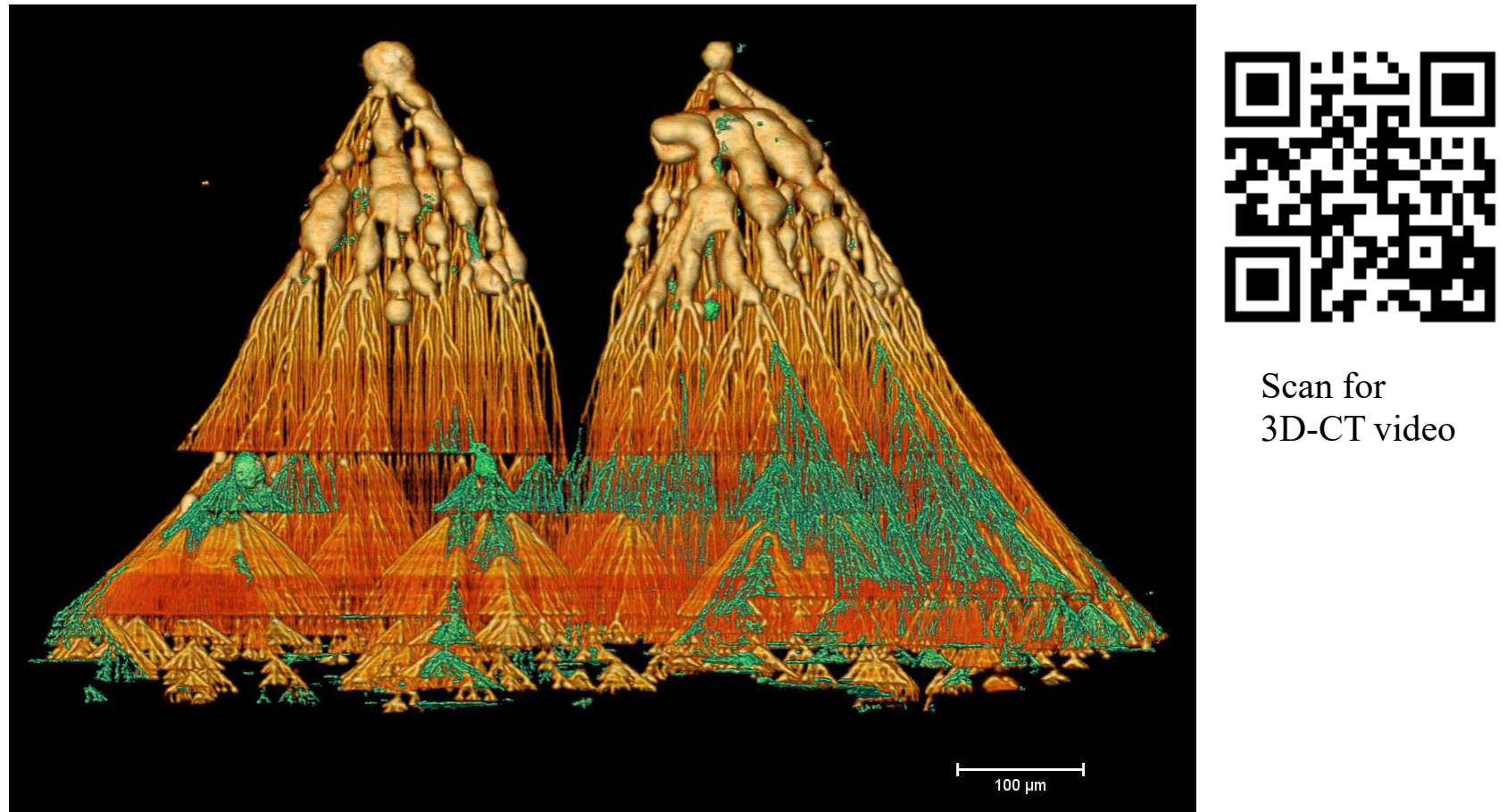

Scan for 3D-CT video

Figure 2. 3D-CT image of hibonite dendrites; crystal face is the lower surface. 